\title{
Effect of Awareness among Smokers and Effect of Smoking on Tear Film in Central India
}

\section{Madan P*, Sune P and Sune M}

Department of Ophthalmology, Acharya Vinoba Bhave Rural Hospital, India

*Corresponding author: Piyush Madan, Postgraduate Student, Department of Ophthalmology Acharya Vinoba Bhave Rural Hospital, Plot 266 Satnami Nagar, Behind Vmv College , CA Road, Nagpur-440008, Sawangi, Wardha Maharashtra, India, Tel: 91-8087896559; E-mail: pmadan01@gmail.com

\section{Abstract}

Introduction: In this study, we studied ocular morbidity among smokers in central India, awareness regarding ocular side effect among smoker and effect of awareness about smoking among smokers. Study was conducted with aim: To study effect of smoking on tear film among smokers in Central India, To study awareness regarding health related problems effect among smokers, To study effect of awareness about smoking among smokers.

Methods: Patient attending ophthalmology OPD with history of smoking cigarettes every day or more than 4 days per week, number of cigarettes - 4 or more per day, duration of smoking - more than 6 months were included in the study. Patients were asked about the complaints and were given questionnaire to see awareness regarding ocular side. Patients were followed in eye OPD with their relative for 6 months to see effect of awareness about smoking among smokers.

Results: The most common symptom found was irritation of eyes seen in 48\%, followed by blurring of vision $16 \%$, burning sensation 15\%, and itching 6\%. Most number of patients had cataract 64\% followed by Dry eye 55\%, ARMD (Age Related Macular Degeneration) 19\%, Glaucoma 9\% and Graves's ophthalmopathy 1.7\%. Only $12 \%$ of subjects were aware of the association between blindness and smoking, the lowest among the six conditions included in the survey.

Conclusion: The awareness of the risk of blindness from smoking is low amongst smokers. Public eye health campaigns viz advertisement, health camp is an important mean of spreading message to smokers to quit smoking.

Keywords: Glaucoma; Age-Related Macular Degeneration; Dry eye; Graves' ophthalmopathy

\section{Abbreviations: ARMD: Age Related Macular Introduction} Degeneration; TBUT: Tear film breakup time.

Cigarette or tobacco smoking is a well-recognised major risk factor for a wide range of diseases, such as 


\section{Open Access Journal of Ophthalmology}

cardiovascular, respiratory, and malignant diseases, in both men and women. Smoking also has adverse ocular effects [1]. In recent years, blindness has emerged as an important cause of smoking-related disability. Notably, smoking has been linked to age-related eye diseases such as cataract, Graves 'ophthalmopathy, glaucoma, and agerelated macular degeneration, two leading causes of visual impairment and blindness globally. Many of these diseases lead to irreversible blindness [2,3]. There is also evidence for a dose-response effect of smoking on eye morbidity. However, relative to other better known smoking-related conditions, the association between smoking and eye diseases is less widely recognised. The awareness of the risk of blindness from smoking is low [4].

Tobacco smoke contains numerous substances, many of which exert toxic influence by producing the ischemia and oxidative stress on various organs including the eye [5]. It leads to the production of toxins which lead to a decreased blood flow to the capillaries and the resultant decrease in nutrition to the eye [6]. Free radicals produced due to smoking lead to impaired cell function [7]. The relationship between smoking and dry eye has not been studied extensively, but for those with dry eye, smoking is a significant irritant that leads to symptoms such as scratchiness, foreign body sensation, burning of the eyes and grittiness. These symptoms are suggestive of dry eye syndrome, and are caused by dysfunction in the thin precorneal tear film which covers the entire cornea and bulbar conjunctiva $[8,9]$.

Study was conducted with aim: To study effect of smoking on tear film among smokers in Central India, To study awareness regarding health related problems effect among smokers, To study effect of awareness about smoking among smokers [8].

\section{Materials and Methods}

This study is adhered to tenets of declaration of Helsinki and was approved by institutional ethical committee of DMIMSU. Informed consent was obtained from all subjects after nature and possible consequences of study were explained to them. The study was conducted in the department of ophthalmology AVBRH attached to JN medical college Sawangi, Wardha, Maharashtra. Study design was Questionnaire based prospective study. It was conducted for 1 year with sample size-170 patients. These patients were explained about the purpose of study before subjecting to questionnaire and performing ocular examination on the examination schedule. Patients were made aware about ill effect of smoking and asked to quit smoking to prevent further ocular damage. All patient attending ophthalmology OPD AVBRH and who met the inclusion criteria were counselled regarding the disease and study those willing patient were selected for study. Informed and written consent was taken from all patients.

- Inclusion criteria: Age - 21-50 years, current smokers smoking cigarettes every day or more than 4 days per week, number of cigarettes - 4 or more per day, duration of smoking more than 6 months.

- Exclusion criteria: People who are not able to give informed consent or complete questionnaire, patient who had known systemic or ophthalmic disease, people using contact lens, any history of long term topical medication, previous ocular surgery.

Questionnaire were prepared and given to patient with the aim to answer the smoking behaviour, ocular and systemic health related complaint and patients perception of effect of smoking on eye. A complete through ophthalmic examination was done.

- Questionnaire: Details of smoking; duration of smoking; number of cigarettes; ocular manifestation of participants.

Their awareness of link between smoking and for smoking attributable conditions (lung cancer, heart disease, stroke and blindness and distracter condition deafness).

All patients were asked to come after 1 week, 1 month, 3 month, and 6 months. Patients were asked about quitting of smoking with their relative in OPD.

\section{Result}

Of the total 170 cases, there were 163 males and 7 females (Figure 2). The age range was between 18 and 50 years. Mean age among males was $34.68 \pm 4.56$ years (2050 ) and females were $32.76 \pm 3.87$ years $(20-50)$ (Figures $1 \& 2)$. 


\section{Open Access Journal of Ophthalmology}

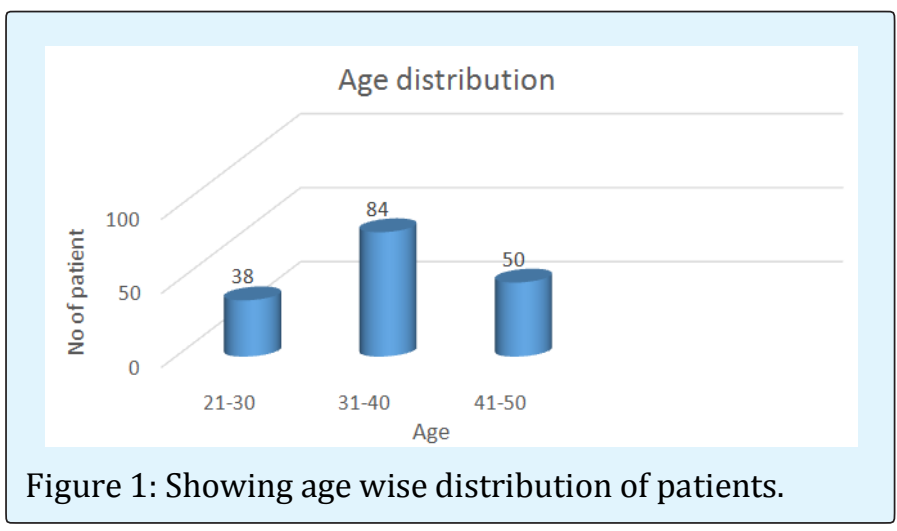

\section{Sex distribution}

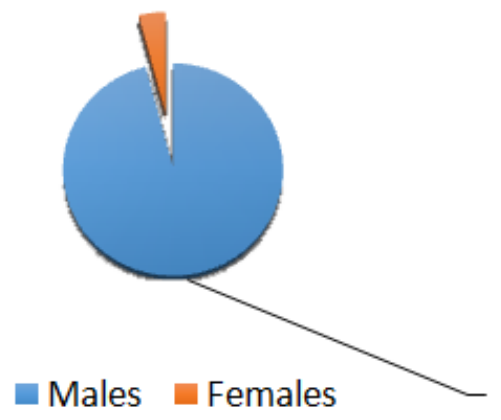

Figure 2: Showing sex distribution of sample.

The ocular symptoms were seen in 146. Of these 146 patients, $91(62.3 \%)$ had irritation of eyes followed by burning sensation in 38 (26\%), and itching in 17 (11.6\%) patients (Figure 3).

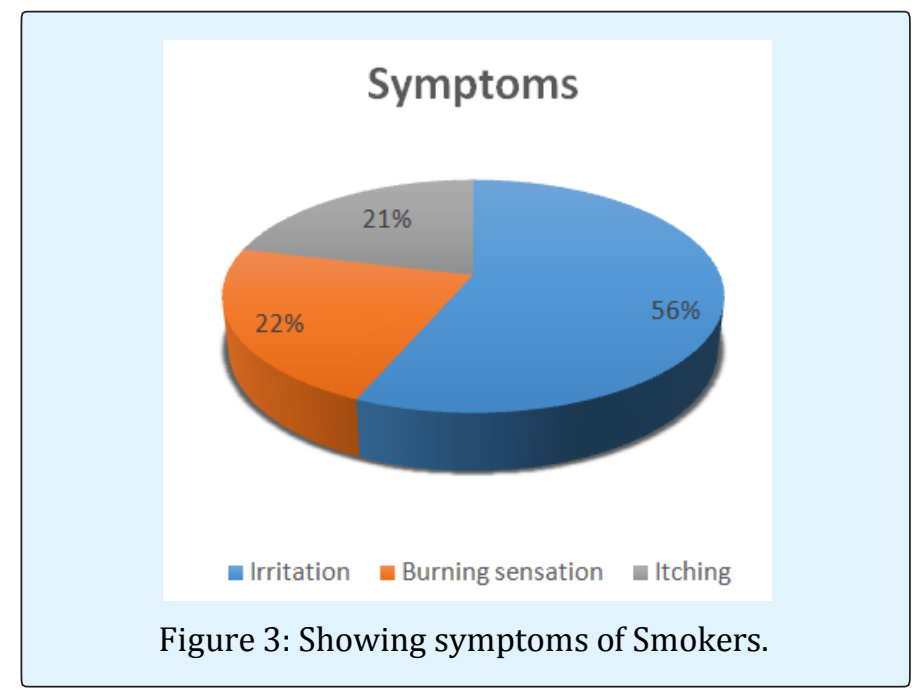

Most patients were aware about lung cancer (93\%), heart attack (85\%), only $12 \%$ patient were aware about blindness due to smoking (Figure 4).

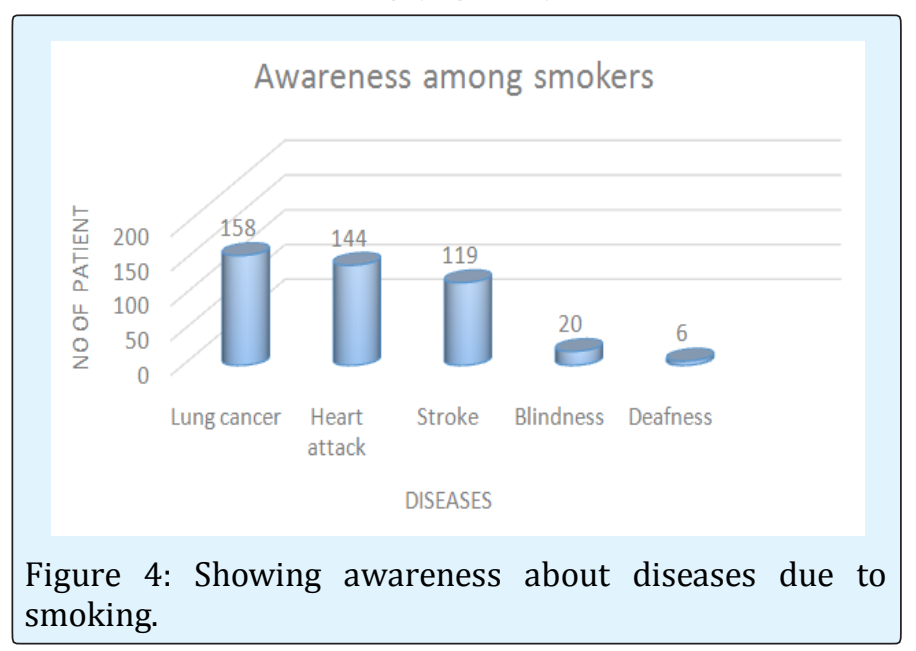

Most number of patients had dry eyes $=146(85.8 \%)$ while $24(14.1 \%)$ didn't have any signs of dry eye.

In our study, dry eye evaluation was done with Schirmer's test 1, Tear film breakup time (TBUT) and Staining of the cornea with fluorescein dye. The first test done was Schirmer's 1 test, where a Schirmer's strip (Whatman no 41 filter paper $40 \mathrm{~mm}$ long, $5 \mathrm{~mm}$ breath) was placed in both the lower eyelid at medial one-third and lateral two third of lower lid and participants were asked to look straight. After $5 \mathrm{~min}$ the readings were taken and recorded. $10 \%$ were diagnosed with severe dry eye, $20 \%$ with moderate dry eye and $25 \%$ with the mild dry eye (Figure 5).

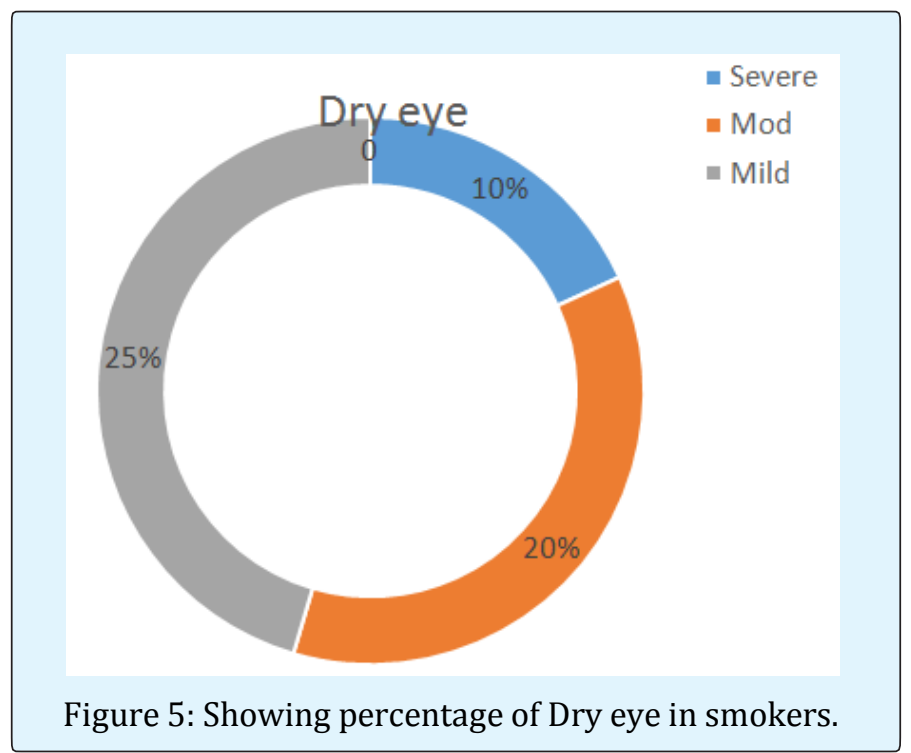




\section{Open Access Journal of Ophthalmology}

Out of 170 only $60(35.29 \%)$ patients quit smoking at end of 6 months. Of these 60 patients, $10(16.66 \%)$ quit smoking at 1 month, 26 (43.33\%) quit smoking at 3 months and $24(40 \%)$ quit smoking at 6 months (Figure $6)$.

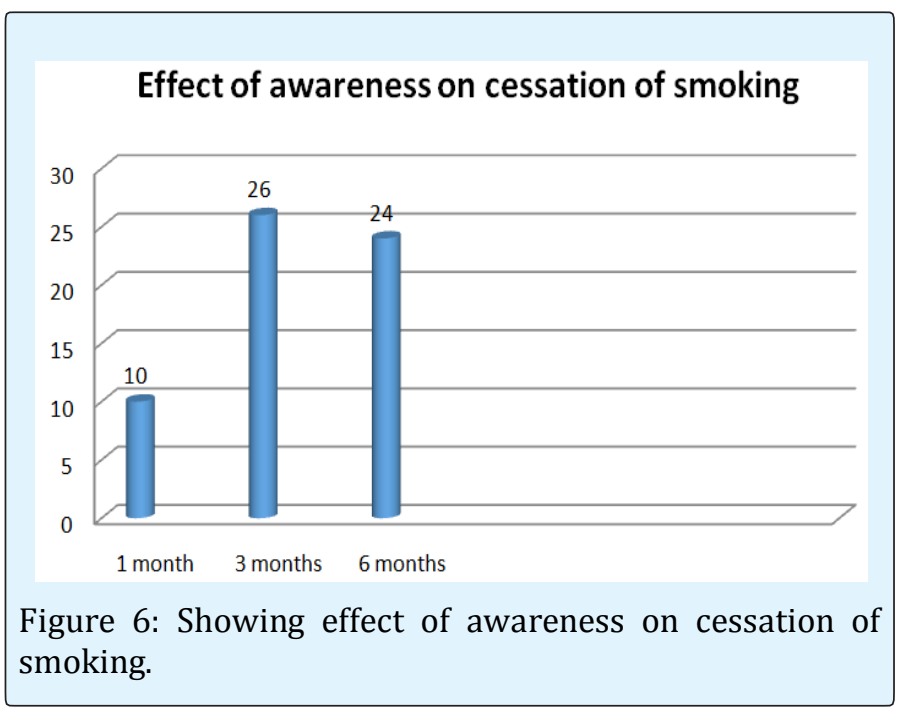

\section{Discussion}

The results of the current study suggest that the awareness of blindness as a smoking-related condition was low among current smokers with ocular conditions attending an ophthalmology clinic. Only $12 \%$ of subjects were aware of the association between blindness and smoking, the lowest among the six conditions included in the survey.

Relationships between dry eye and smoking have been studied previously. Smokers are known to have symptoms of irritation, burning, watering, and foreign body suggestive of dry eye. In our study irritation of eyes was seen in 91 (62.3\%), followed by burning sensation 38 (26\%), and itching $17(11.6 \%)$. Smoking causes a dysfunctional tear film, which results in dry eye. The mechanism by which smoking causes pre-corneal tear film breakdown has not been confirmed, but the presumptive cause is the effect of lipid peroxidation of the outer pre-corneal tear film. Studies have reported damage in lipid layer, which prevent even spreading of tear film. Smokers are reported to have increased lipid peroxidation. Cigarette smoke contains free radicals, aldehyde, peroxidases, epoxides, nitrous oxides, and other pro oxides, these oxidant groups contribute to the damage [10].
Chronic smoking has been reported to cause quantitative and qualitative disturbance in ocular surface health.

Our finding was similar to that in another study on adult outpatients in ophthalmic, general medical, and general surgical clinics in Singapore and Scotland, in which the awareness of blindness as a smoking-related condition was $36.5 \%$ and $30.5 \%$, respectively, the lowest amongst a list of conditions including lung cancer, mouth and throat cancer, heart disease, and stroke $[10,11]$. Awareness of a smoking-related disease may help in reducing the number of cigarettes smoked but this may also be dependent on the perceptions of vulnerability by the smokers and the extent to which they consider the condition to be life threatening. Health concerns remain the primary motive for smoking cessation [10]. Smokers who believe that they have a smoking-related disease and attribute their symptoms to smoking are more motivated to quit [12,13]. In addition, smokers who have experienced and attributed an acute sentinel health event to smoking were more likely to stop smoking compared with community-based estimates [14].

About 93\% of South-Korean men hospitalised with cardiovascular disease reported their intention to quit smoking [15]. It was also found that smokers who attributed respiratory symptoms to smoking were more likely to quit smoking than others [13]. The current study showed that heart attack was the greatest source of concern among smokers. Our subjects were less fearful of irreversible central blindness from smoking than potentially life-threatening systemic diseases such as heart attack, lung cancer, and stroke, when they were asked to prioritise treatment and prevention among the six conditions included in the survey.

In our study, overall $35.29 \%$ patients quit smoking at end of 6 months. The majority of smokers in our study had normal or near-normal vision and, therefore, may be less fearful of developing blindness from smoking. This might be suggestive of a possible barrier towards smoking cessation in those who are poorly motivated. As such, it may be more appropriate to offer further education on effects of smoking before encouraging a cessation attempt. Low percentage of patients quitting smoking may also be attributed to level of educations.

Our study in an Asian population yielded results similar to that of Bidwell, et al. and Moradi, et al. in that the motivation score to quit smoking due to irreversible central blindness was the second highest among all the 


\section{Open Access Journal of Ophthalmology}

conditions examined, following only lung cancer $[16,17]$. Hence, despite a low level of awareness, blindness is still a significant motivational factor for smoking cessation. In our study all patients had normal best corrected visual acuity which is also one of the reasons less number of patients quit smoking.

\section{Conclusion}

Effect of smoking was severe on tear film because 146 patients (85.6\%) were diagnosed of dry eye.

The awareness of the risk of blindness from smoking is low amongst smokers. The fear of blindness may be as powerful a motivational factor to quit smoking as fear of non-ophthalmic smoking-related diseases.

This study assesses the awareness of blindness and other smoking-related conditions on a potentially at risk population of current smokers attending an ophthalmology outpatient clinic. Currently, no data exists on such a large sample of current smokers with eye diseases. This vulnerable group was found to be less fearful of irreversible central blindness from smoking than potentially life threatening systemic diseases such as heart attack, lung cancer, and stroke. Of the smokingrelated diseases studied, the motivation to quit smoking due to irreversible central blindness was the second highest after lung cancer.

The ill effects of smoking should be stressed by public eye health campaigns done by the government as well as private health organizations to encourage people to stop smoking. This indicates the lack of eye health promotion information in regard to smoking. We suggest, adverse effects of smoking on the eye should be stressed to younger age groups, which are more prone for addictions as majority of smokers start at an early age.

\section{Limitations}

First, the symptoms are self-reported and may differ from objective clinical measurement. A control group was not taken in this study. The participants might have been subjected to external factors like exposure at work, passive smoking, air pollution, etc., may affect the eye and is not evaluated. The quantity of tobacco used was not measured and compared with the severity of dry eye. There is also an external social desirability factor bias in a country like India, which could cause smokers to report reduced number of cigarettes smoked. Finally, a hospital sample may not represent general population of the locality.

\section{References}

1. Bartecchi CE, MacKenzie TD, Schrier RW (1994) The human costs of tobacco use. N Engl J Med 330(13): 907-912.

2. Solberg Y, Rosner M, Belkin M (1998) The association between cigarette smoking and ocular diseases. Surv Ophthalmol 42(6): 535-547.

3. Woo JH, Au Eong KG (2008) Don't lose sight of agerelated macular degeneration: the need for increased awareness in Singapore. Singapore Med J 49(11): 850-853.

4. Vingerling J, Hofman A, Grobbee D, DeJong P (1996) Age-related macular degeneration and smoking. The Rotterdam Study. Arch Ophthalmo 114(10): 11931196.

5. Handa S, Au Eong KG (2006) Time to raise awareness of blindness as another smoking-related condition. Ann Acad Med Singapore 35(5): 379-380.

6. Gayton JL (2009) Etiology prevalence and treatment of dry eye disease. Clin Ophthalmol 3(14): 405-412.

7. Owusu-Dabo E, Lewis S, McNeill A, Gilmore A, Britton J (2011) Support for smoke-free policy, and awareness of tobacco health effects and use of smoking cessation therapy in a developing country. BMC Public Health 18(11): 572.

8. Altinors DD, Akca S, Akova YA, Bilezicki B, Goto E, et al. (2006) Smoking associated with damage to the lipid layer of the ocular surface. Am J Ophthalmol 141(6): 1016-1021.

9. Matsumoto Y, Dogru M, Goto E, Sasaki Y, Inoue H, et al. (2008) Alterations in tear film and ocular surface health in chronic smokers. Eye 22(7): 961-968.

10. Thomas J, Jacob GP, Abraham L, Noushad B (2012) The effect of smoking on the ocular surface and the precorneal tear film. Australas Med J 5(4): 221-226.

11. Ng DHL, Roxburgh STD, Sanjay S, Au Eong KG (2010) Awareness of smoking risks and attitudes towards graphic health warning labels on cigarette packs: a 


\section{Open Access Journal of Ophthalmology}

cross-cultural study of two populations in Singapore and Scotland. Eye 24(5): 864-868.

12. Coleman T, Barrett S, Wynn A, Wilson A (2003) Comparison of the smoking behaviour and attitudes of smokers who believe they have smoking-related problems with those who do not. Fam Pract 20(5): 520-523.

13. Walters N, Coleman $T$ (2002) Comparison of the smoking behaviour and attitudes of smokers who attribute respiratory symptoms to smoking with those who do not. Br J Gen Pract 52(475): 132-134.

14. Boudreaux ED, Baumann BM, Camargo CAJ, O'Hea E, Ziedonis DM (2007) Changes in smoking associated with an acute health event: theoretical and practical implications. Ann Behav Med 339(20): 189-199.
15. Sohn M, Stotts NA, Benowitz N, Christopherson D, Kim KS, et al. (2007) Beliefs about health, smoking, and future smoking cessation among South Korean men hospitalized for cardiovascular disease. Heart Lung 369(5): 339-347.

16. Bidwell G, Sahu A, Edwards R, Harrison RA, Thornton J, et al. (2005) Perceptions of blindness related to smoking: a hospital-based cross-sectional study. Eye 19(9): 945-948.

17. Moradi P, Thornton J, Edwards R, Harrison RA, Washington SJ, et al. (2007) Teenagers' perceptions of blindness related to smoking: a novel message to a vulnerable group. Br J Ophthalmol 91(5): 605-607. 